\title{
MONOGRAPHIE DU GENRE THREMMA
}

\author{
Trichoptera, Thremmatidae
}

par J. GIUdicell, ${ }^{*}$.

Le genre Thremma est représenté par trois espèces européennes. Thremma anomalum Mc Lachlan, espèce d'Europe centrale, et Thremma gallicum Mc Lachlan, espèce d'Europe occidentale, sont connues à tous les stades de leur développement. KLApalek a décrit en 1908 la larve et la nymphe de $T$. gallicum et, plus récemment, cette espèce a fait l'objet d'une étude morphologique plus complète (JAcquemart et Coineau, 1966). On doit à Botosaneanu (1956) une excellente description de $T$. anomalum à l'état larvaire et nymphal. Quant à la troisième espèce, Thremma sardoum Costa, son aire de répartition se limite à la Corse et à la Sardaigne; jusqu'à présent, seul l'imago était connu, surtout grâce à Moses.y (1930) qui a donné la première description des genilalia.

Dans la présente étude, je signale l'existence de deux lignées divergentes au sein de l'espèce $T$. gallicum. L'une correspond à la population des Pyrénées, l'autre à celle du Massif Central. Je propose de placer celte dernière population dans une nouvelle sous-espèce avec l'appellation de Thremma gallicum arvernense n. ssp. En outre, je complète la connaissance de $T$. sardoum en insistant plus particulièrement sur la morphologie larvaire.

\section{1. - Thremma gallicum Mc Lachlan et Thremma gallicum arver- nense n. ssp.}

En France continentale, on ne trouve que l'espèce Thremma gallicum, localisée dans les Pyrénées et dans le Massif Central. Grâce à l'amabilité de mon collègue H. DÉcamps, j'ai pu disposer d'un abondant matériel en provenance des Pyrénées, tandis que des récoltes dans un complexe de sources rhéocrènes situé près du village de Lanarce (Ardèche), sur la bordure méridionale du Massif Central, m'ont fourni de nombreux exemplaires de Thremma.

1. Université de Provence. Laboratoire de Biologie Animale (Ecologie). Centre de Saint-Jérôme. Traverse de la Barasse, 13-Marseille 13e. 
L'examen comparatif des individus provenant des Pyrénées et du Massif Central fait apparaître des différences morphologiques entre les deux populations. Ces différences sont constantes et se manifestent au niveau des genitalia mâles. Elles sont représentées dans les figures 1 et 2 . Je les énumère ci-dessous.
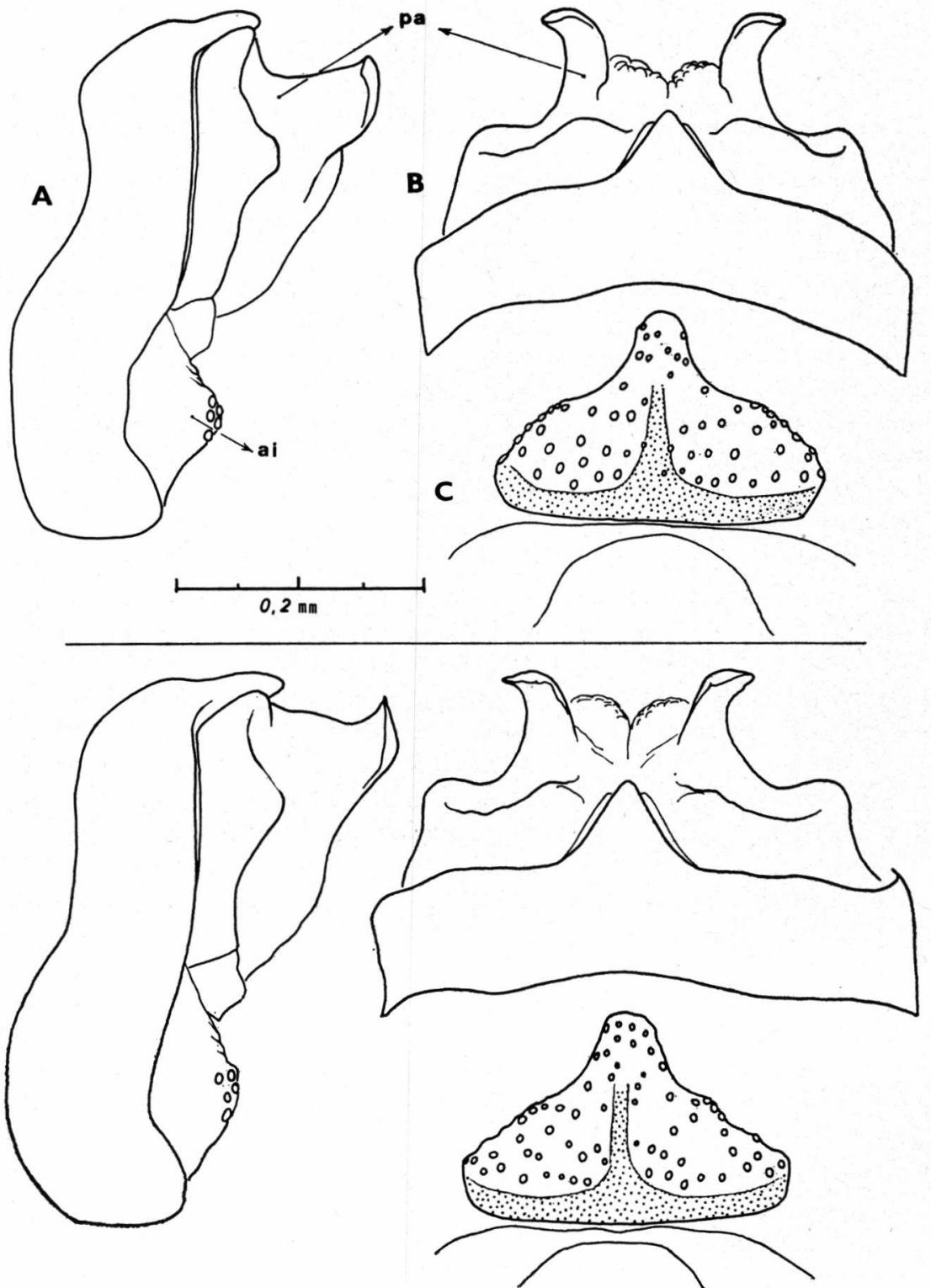

FIG. 1. - Thremma gallicum gallicum. Genitalia mâles. - A : Vue latérale;

B : Vue dorsale; C : Vue ventrale du' $10 \mathrm{e}$ segment et de la plaque ventrale - $p a$ : parapoctes; $a i$ : appendices inférieurs. 

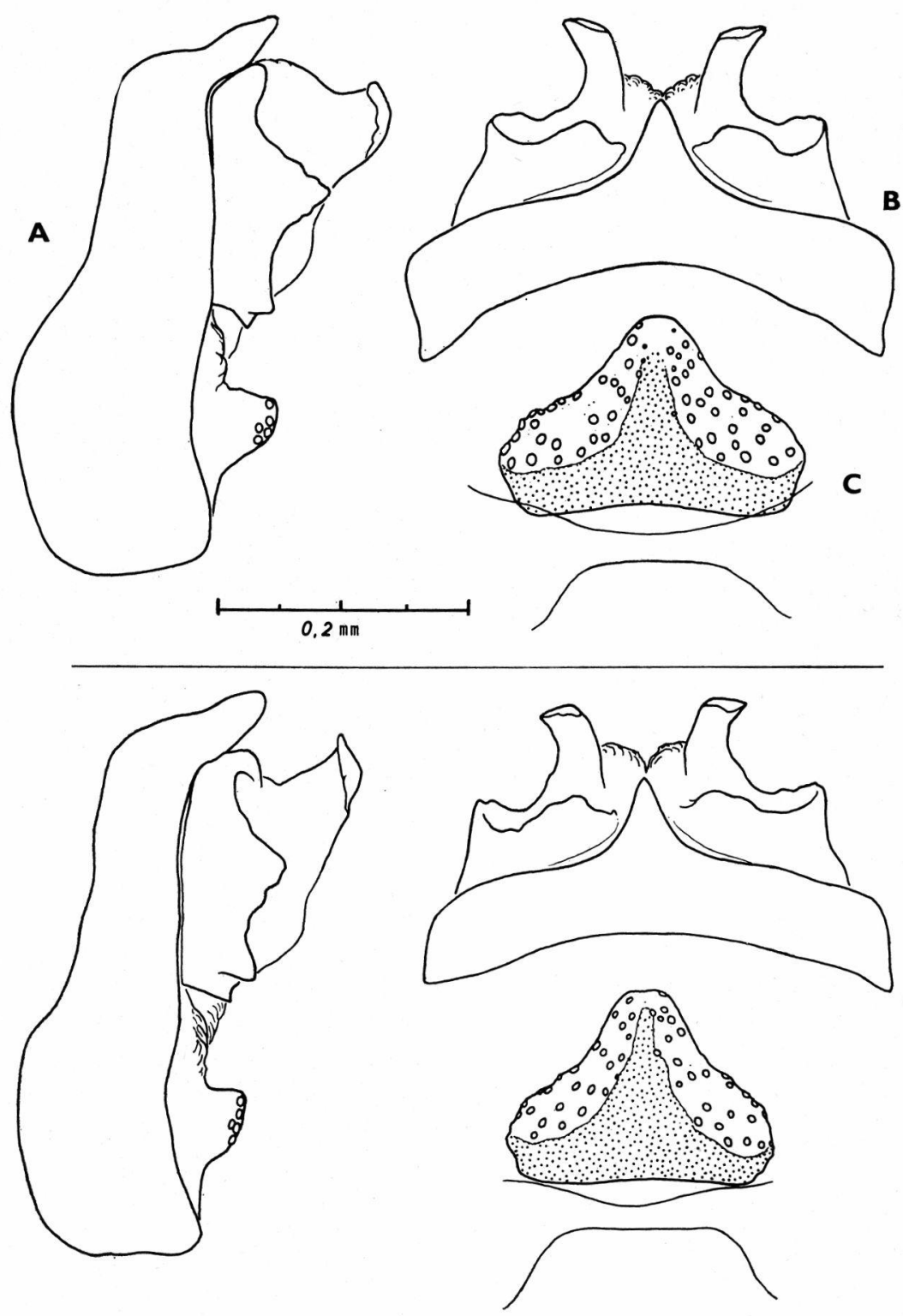

FIg. 2. - Thremma gallicum arvernense. Genitalia mâles. 
- $1^{\circ}$ Dorsalement, le bord postérieur du $10^{\circ}$ segment abdominal se prolonge en arrière en une pointe plus aiguë chez la forme du Massif Central que chez la forme pyrénéenne.

- $2^{\circ}$ Ventralement, le $10^{\circ}$ segment est réduit à une bande sclérifiée, plus large chez les exemplaires du Massif Central.

- $3^{\circ}$ La forme de la plaque ventrale diffère d'une population à l'autre; l'extrémité postérieure de cette plaque est plus large et son aire pigmentée (zone ponctuée sur le dessin) est plus étendue chez les individus du Massif Central.

- $4^{\circ}$ Les appendices intermédiaires (paraproctes), en vue latérale, sont plus larges et moins longs chez les individus du Massif Central.

- 5" Les appendices inférieurs (a.i.) sont saillants chez la forme du Massif Central; ils ont une base très large et sont peu différenciés chez la forme pyrénéenne.

Je n'ai observé aucune différence entre les deux populations au niveau du penis et dans la coloration des imagos.

Les caractères distinctifs, exposés ci-dessus, sont constants et nets; cependant, ils ne sont pas très accusés. Je considère que la divergence morphologique entre les deux populations est du niveau de la sous-espèce. On peut supposer que cetle divergence s'est établie après la séparation du Massif Central et des Pyrénées, c'està-dire après fragmentation d'une aire qui était continue à la période hercynienne.

Mc. LACHLAN (1884), lorsqu'il réalisa la description originale de T. gallicum, disposait de deux mâles de l'espèce, l'un provenant des Pyrénées (vallon de Burbe, près de Luchon, Haute-Garonne), l'autre provenant du Massif Central (Le Livran, Cantal). Le dessin des genitalia mâles que donne Mc. LAchlan (planche LVII) est très sommaire et n'offre aucun caractère permettant d'attribuer à l'une ou à l'autre des deux populations géographiques l'exemplaire ayant servi à décrire l'espèce.

Dans la description originale, l'exemplaire des Pyrénées est cité en premier; on peut donc le considérer comme le lectotype de Thremma gallicum ${ }^{1}$. Je suis amené alors à considérer les exemplaires du Massif Central comme représentant une sous-espèce que je dénomme Thremma gallicum arvernense.

\section{2. - Thremma sardoum Costa.}

On ne trouve aucune mention des genitalia de l'espèce ni dans la description originale de Costa (1884), ni dans l'ouvrage de Mc. Lachlan (1884). On doit à Mosery (1930) la seule description et la seule représentation des genitalia; elles permettent une détermination sûre de l'espèce.

1. «Si une espèce nominale n'a pas d'holotype, tout zoologiste peut désigner un des syntypes comme lectotype ». (Code international de nomenclature zoologique, Londres, 1961). 
Je profite de la présente étude pour apporter une iconographie plus précise et aussi plus complète des genitalia (cf. fig. 3 ).

Les caractères les plus remarquables portent sur la forme de la plaque ventrale et sur le pénis; ce sont d'ailleurs ces pièces génitales qui distinguent le mieux les imagos des trois espèces de Thremma ${ }^{1}$.
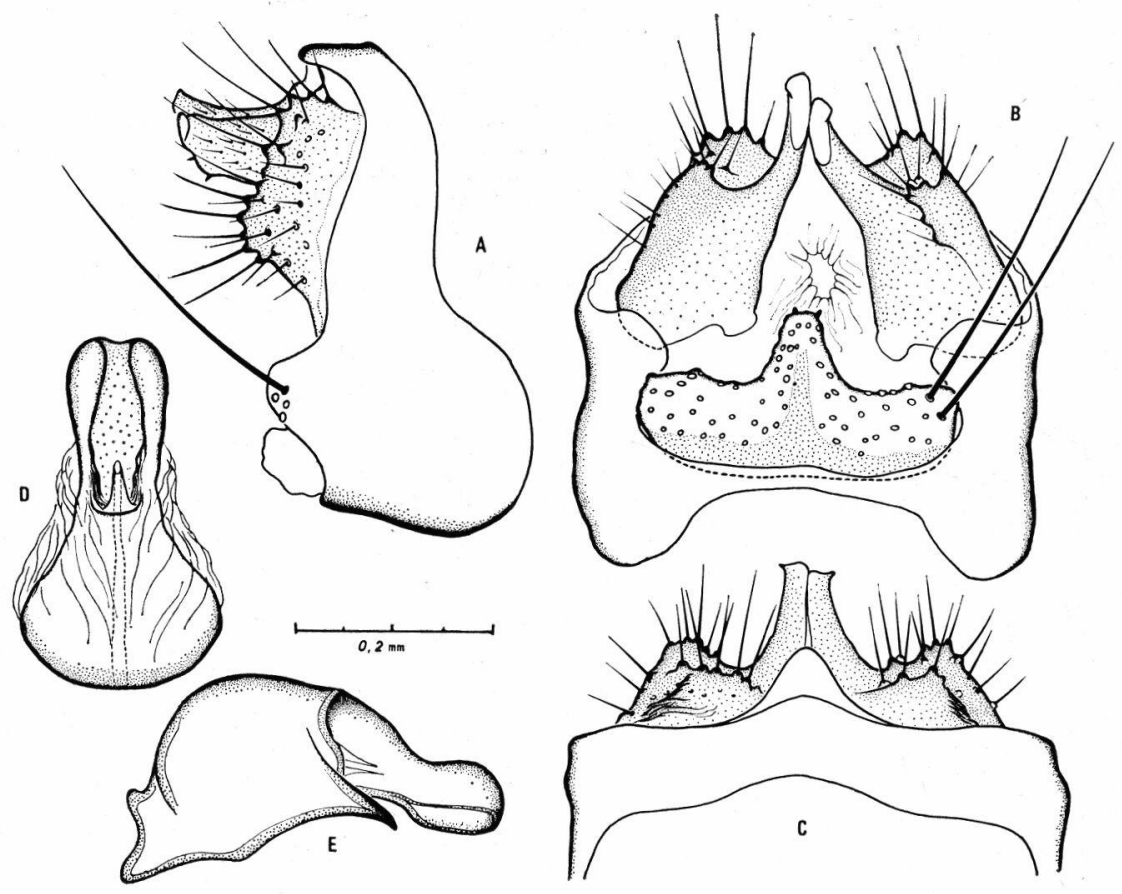

Fig. 3. - Thremma sardoum. Genitalia mâles. - A : Vue latérale; B : Vue ventrale; C : Vue dorsale; D : Pénis, vue dorsale; E : Pénis, vue latérale.

\section{DESCRIPTION DE LA LARVE :}

La larve présente l'habitus caractéristique des Thremma, avec la tête rabattue ventralement sous le pronotum et invisible du côté dorsal, avec la sclérification originale du mesonotum, l'abdomen trapu et conique, la présence d'un mamelon ventral très saillant sur le premier segment abdominal (cf. fig. 4 et 5).

Je ne veux pas entreprendre ici une description détaillée de la larve de $T$. sardoum car cela me conduirait à donner en même

1. On trouvera un bon dessin de la plaque ventrale de $T$. anomalum in Botosaneanu, 1957. 


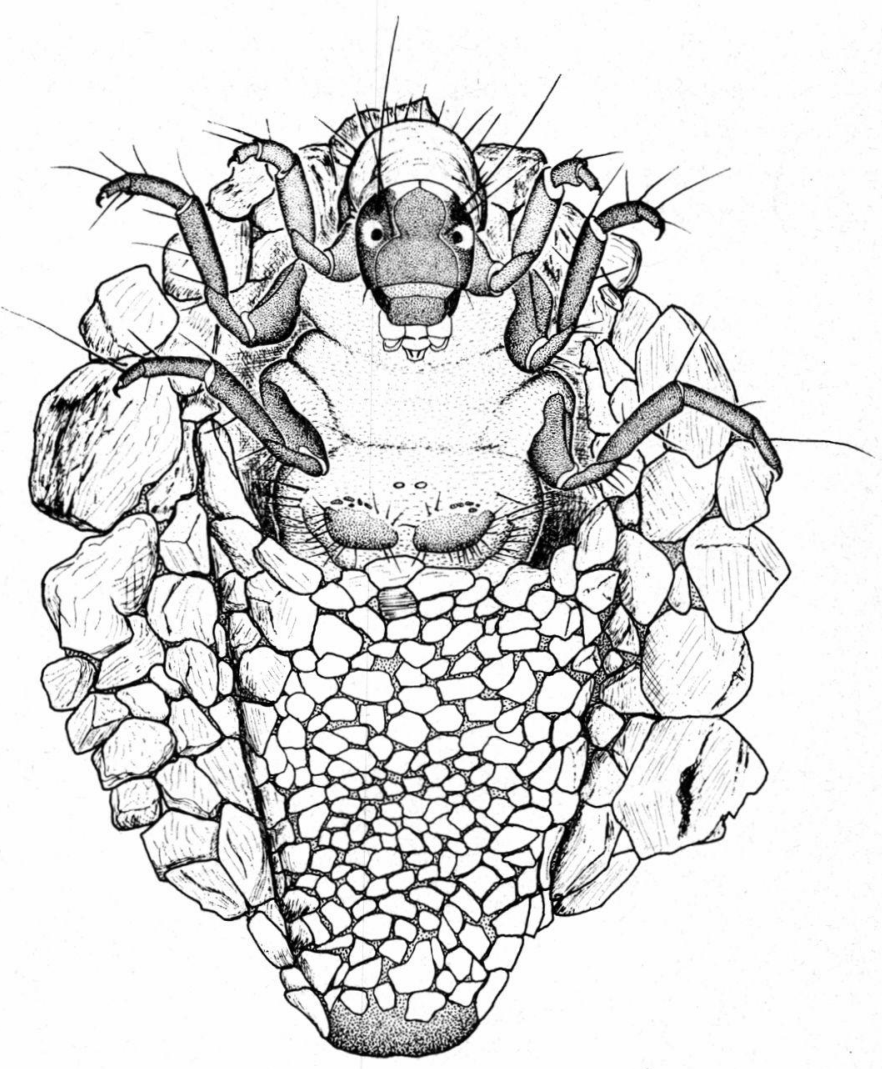

FIG. 4. - Thremma sardoum. Habitus de la larve.

temps une nouvelle description du genre, en raison de la constance des caractères larvaires d'une espèce à l'autre. Or, une telle description existe déjà dans le travail de Botosaneanu (1956) sur Ia larve de $T$. anomalum.

Pour T. sardoum, je me bornerai à signaler les caractères spécifiques originaux.

Longueur de la larve au dernier stade : 5,4 à $5,7 \mathrm{~mm}$.

La couleur générale des sclérites céphaliques et thoraciques est d'un brun plutôt clair.

Capsule céphalique presque aussi large que longue. Dorsalement, la capsule céphalique est brune; frontoclypeus uniformément pigmenté; zones circumoculaires claires. Dans la région occipitale, se trouve une grande tache blanche de part et d'autre de la suture postérieure (suture coronale); cette tache est en contact avec le bord postérieur du clypeus et s'étend jusqu'au bord postérieur de la capsule céphalique. Latéralement et ventralement, la pigmentation de la capsule s'éclaircit beaucoup. 


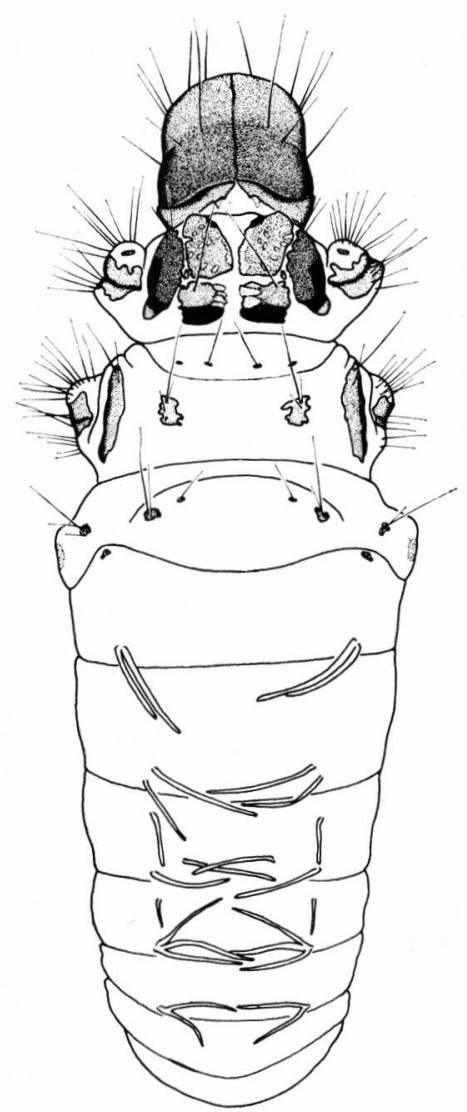

Fig. 5. - Thremma sardoum. Larve au dernier stade; Vue dorsale.

Les mandibules peuvent présenter deux aspects (cf. fig. 6). Certaines sont constituées de deux parties à peu près égales :

- une partie distale, rougeâtre et plus claire, comprenant du côté interne un long tranchant dorsal et un tranchant ventral plus court,

- une partie basale, noire et opaque, sur laquelle se prolonge le tranchant dorsal; sur une courte portion de ce tranchant s'insère un pinceau de 8 à 10 soies plumeuses.

Les mandibules du deuxième type ont leur partie distale réduite. Il existe aussi, entre les deux types précédents, des formes de passage marquées par la réduction plus ou moins importante de la partie distale.

Il apparaît que cette partie de la mandibule se réduit par usure au cours de la vie de la larve; en effet, la réduction est maximale chez les larves les plus âgées.

Les tergites thoraciques ne montrent aucune particularité par rapport aux autres espèces. Notons que chez certains individus peut s'individualiser sur le mésonotum une $4^{\circ}$ paire de petits sclérites entre les 3 paires constantes de gros sclérites; ces deux petits sclérites supplémentaires sem- 


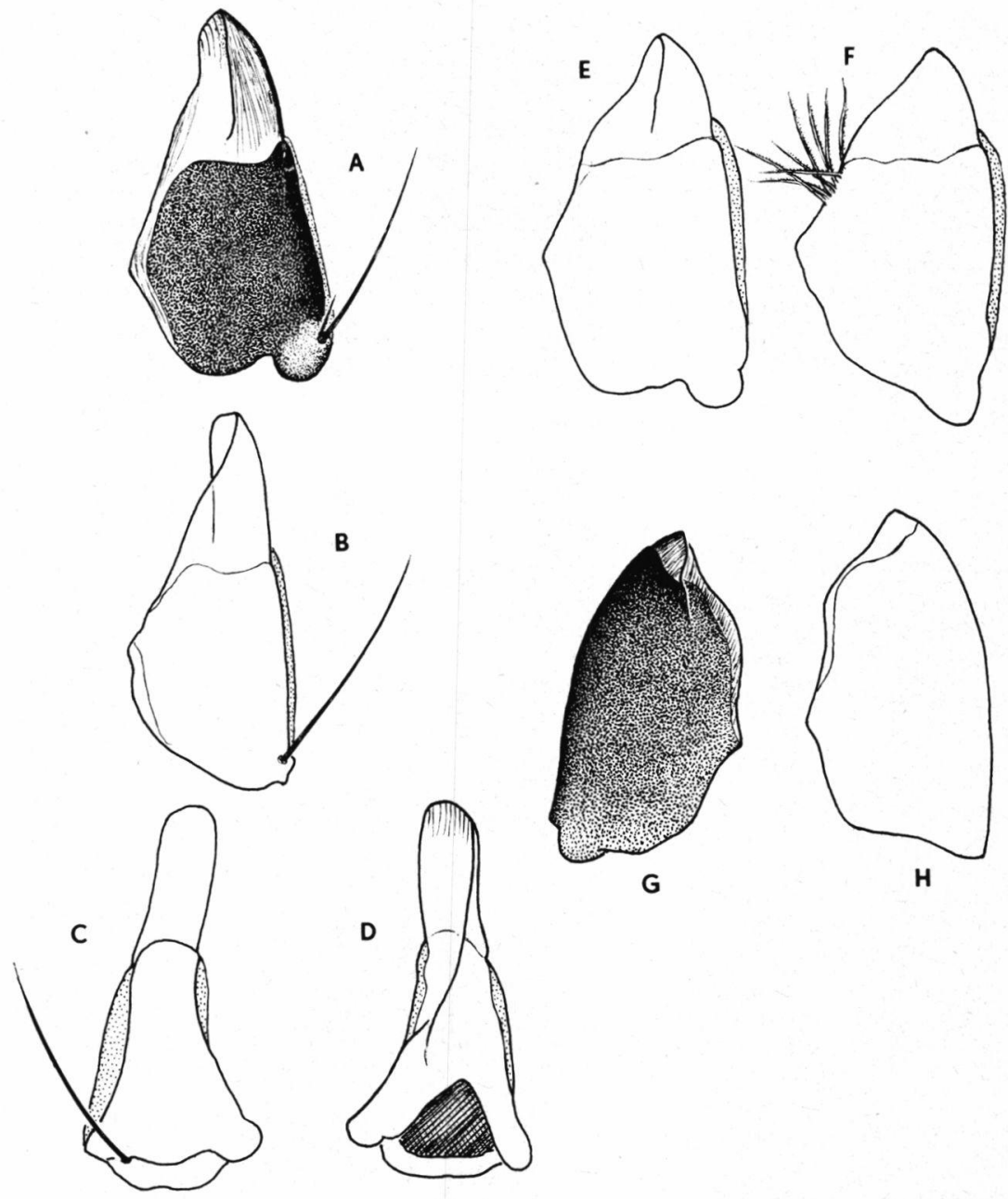

Fig. 6. - Thremma sardoum. Mandibules de la larve, à divers stades d'usure. - A : Mandibule gauche, vue du côté ventral; B : Mandibule droite, côté dorsal; C : Mandibule droite, côté latéral interne; D : Mandibule droite, côté latéral interne; E, F : Mandibules à un stade d'usure moyenne; G, $\mathrm{H}$ : Mandibules au dernier stade d'usure.

blent exister aussi chez certaines larves des deux autres espèces (cf. BOTOSANEANU, 1956).

Le premier segment abdominal possède, comme chez les deux autres espèces un grand mamelon ventral, sorte de tablier pourvu de deux sclérites, débordant largement sur le deuxième segment. 
SChÉma des bRanchies :

\begin{tabular}{ccc}
\hline & V & $\frac{D}{0}$ \\
\hline II & $\frac{0}{3}$ & $\frac{0}{2-3}$ \\
III & $\frac{2}{3}$ & $\frac{0-1}{2-3}$ \\
IV & $\frac{2}{3}$ & $\frac{1-2}{3}$ \\
V & $\frac{1}{2-3}$ & $\frac{1-2}{3}$ \\
VI & $\frac{0-1}{2}$ & $0-1$ \\
VII & $\frac{2}{2}$ & \\
\hline
\end{tabular}

Le fourreau larvaire est bâti à l'aide de grains de sables de tailles variables. Il est du type «ancyliforme », c'est-à-dire qu’il comprend, d'une part, une loge centrale conique et arquée qui contient le corps de la larve, et, d'autre part, deux ailes arrondies, chacune inséréc sur un des côtés de la loge. Le contour de ce fourreau est circulaire ou elliptique, mais les bords ont un aspect déchiqueté car les parties latérales du fourreau sont construites de grains plus gros que ceux de la loge centrale (cf. fig. 7).
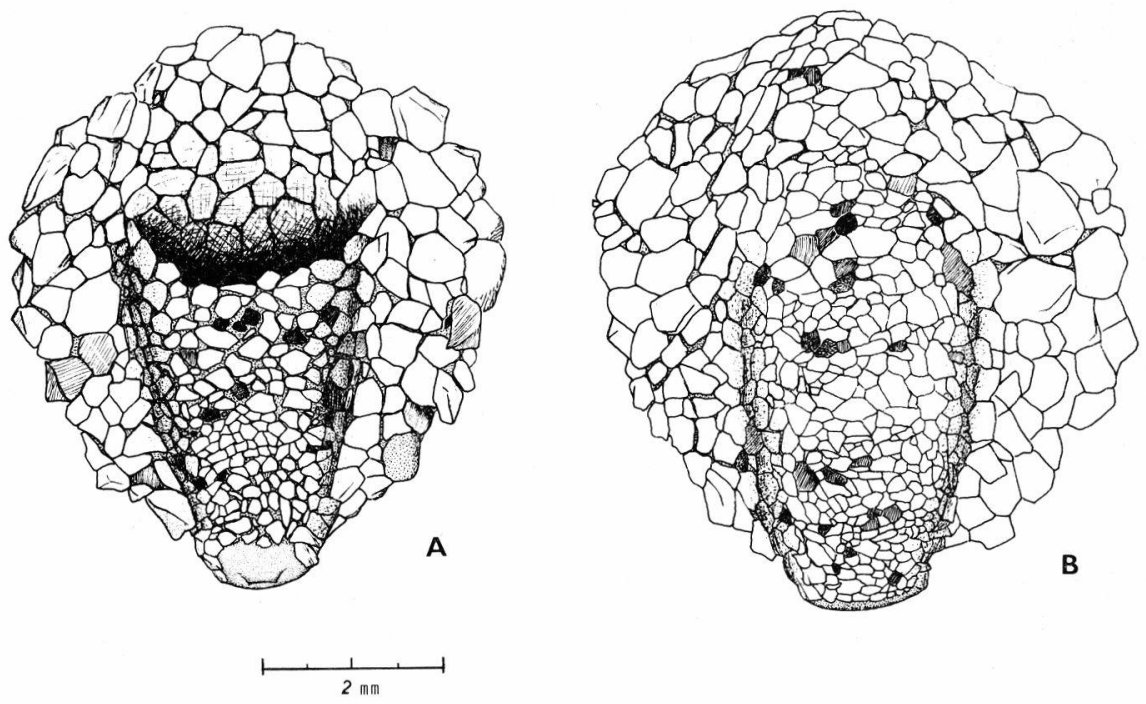

Fig. 7. - Thremma sardoum - Fourreau larvaire. - A: Vue ventrale; B : Vue dorsale. 
Chez les larves très jeunes, les expansions latérales sont très peu développées; elles sont marquées par un simple alignement de grains de sable.

\section{3. - Etude comparée des larves de Thremma.}

Les larves du genre présentent unc assez grande homogénéité morphologique. On note pourtant quelques caractères de différenciation spécifique.

TaIlle : La larve de $T$. anomalum est la plus petite des trois $(4,5 \mathrm{~mm}$ de longueur moyenne et $5,5 \mathrm{~mm}$ pour les deux autres espèces).

Coloration des scLérites: Brun sombre, presque noir pour $T$. anomalum; brun moyen pour $T$. gallicum; brun-roux clair pour T. sardoum.

CAPSULE CÉPHALIQUe : Zone occipitale blanche chez $T$. anomalum et $T$. sardoum; présence de deux grosses taches pigmentées au sein de cette zone claire chez $T$. gallicum.

Mamelon ventral : La forme des deux sclérites varie d'une espèce à l'autre, comme on peut le voir dans la figure 8 .

Fourreau : Il fournit le caractère le plus net pour distinguer facilement les larves des trois espèces (cf. fig. $7,9,10$ ). Le fourreau de $T$. anomalum est simple, conique el recourbé tandis que celuj des deux autres espèces, avec ses grandes expansions latérales, est du type ancyliforme. Chez T. gallicum, il est fait de grains de sable fin; en outre, le bord externe des deux ailes est marqué par un dépôt continu et régulier d'une sécrétion cornée (soie). Chez T. sardoum, le fourreau est construit de grains de sable grossier; son contour est beaucoup plus irrégulier car il n'est pas limité par une sécrétion.

\section{4. - Notes écologiques.}

Les formes aquatiques des Thremma ont été trouvées dans des types d'eaux assez divers. Cependant, il semble que les larves sont caractéristiques du crénon; elles réalisent leur optimum écologique dans le biotope des sources et des ruisselets de sources de la zone prémontane et montane.

Les larves se nourrissent presque exclusivement de Diatomées dont les frustules se retrouvent extrêmement abondantes dans les contenus stomacaux.

Les deux espèces d'Europe continentale ont une large répartition altitudinale : T. gallicum, dans les Pyrénées, a été trouvée entre 500 et $2300 \mathrm{~m}$ d'altitude (DÉCAMPs, 1967) et $T$. anomalum, 

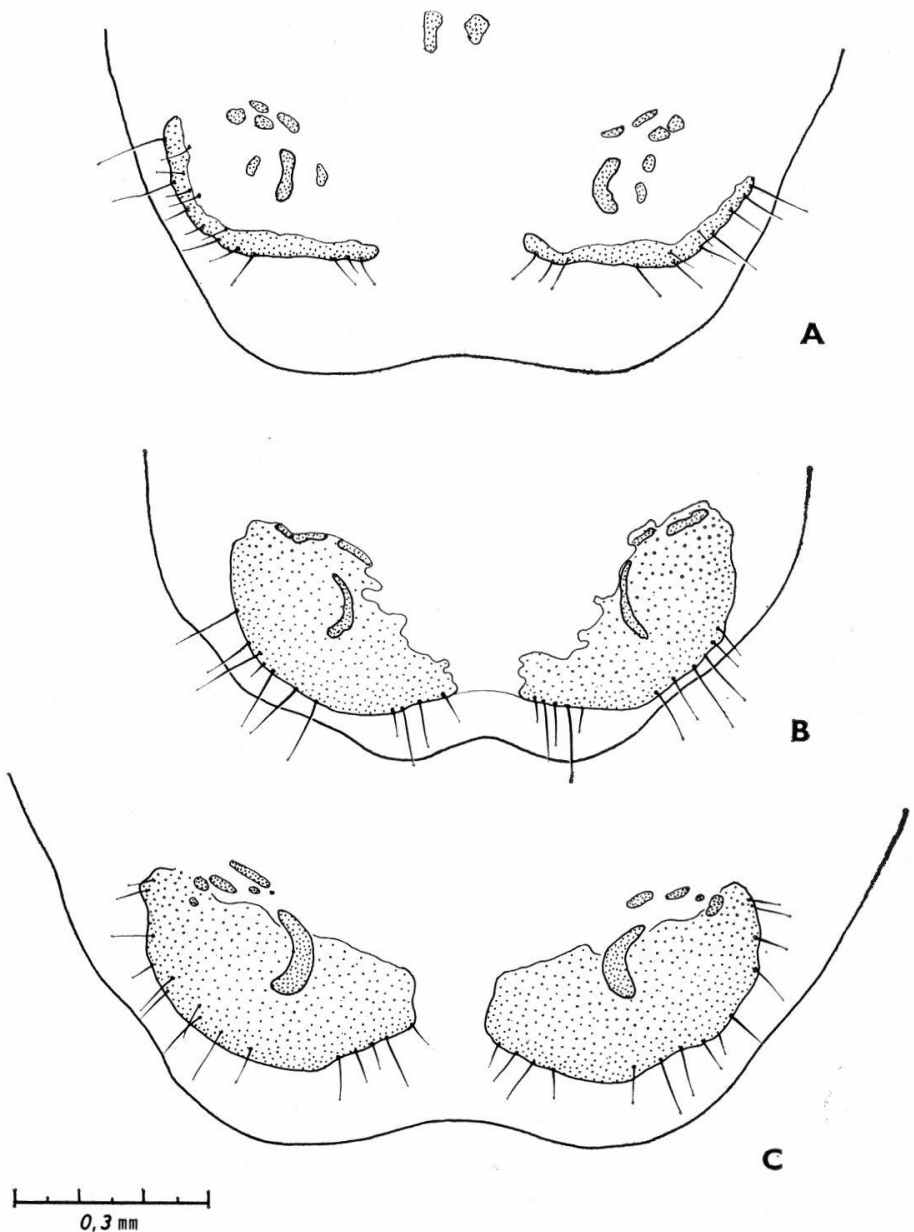

Fig. 8. - Sclérites du mamelon ventral des larves de Thremma - A : T. gallicum; B : T. anomaluin; C : T. sardoum.

dans les Balkans, se situe entre 150 et $2500 \mathrm{~m}$. Par contre, $T$. sardoum en Corse n'atteint pas des altitudes aussi élevées : dans le réseau hydrographique du Tavignano-Restonica, elle s'étage entre 200 et $1250 \mathrm{~m}$ d'altitude (GIUDicelli, 1968).

T. anomalum occupe des biotopes très différents au sein de son aire géographique (Botosaneanu, 1956, 1960). Dans le Massif du Banat (terminaison occidentale des Carpates méridionales), l'espèce est strictement crénobionte : elle se cantonne dans les sources entre 150 et $750 \mathrm{~m}$ d'altitude. Par contre, en Yougoslavie, 


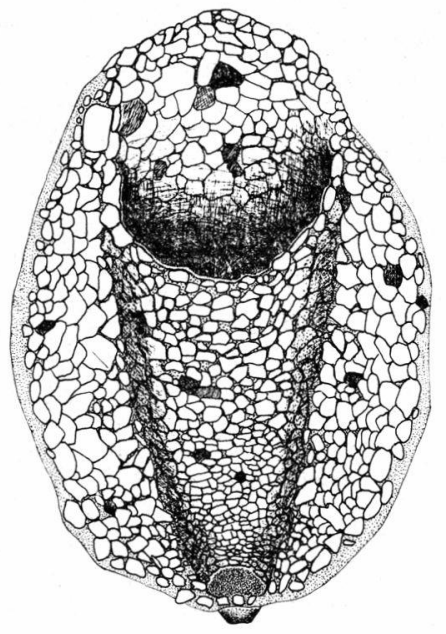

A

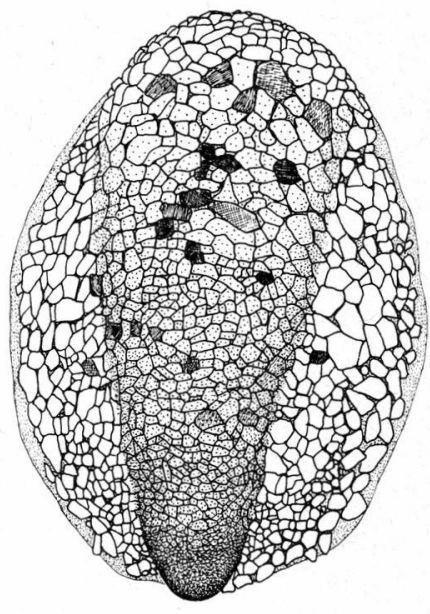

B

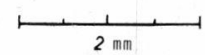

Fig. 9. - Fourreau larvaire de Thremma anomalum - A : Vue ventrale; B : Vue latérale.

is...

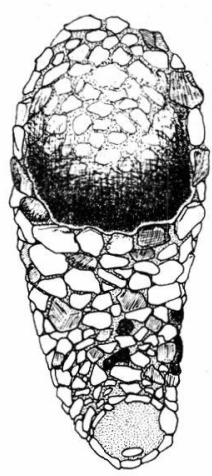

A

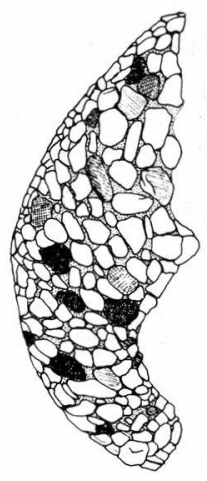

B

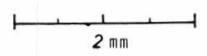

FIG. 10. - Fourreau larvaire de T. gallicum arvernense, - A : Vue ventrale: B : Vue dorsale. 
elle est beaucoup plus alticole : elle vil dans les sources et les torrents froids de montagne, entre 2000 et $2500 \mathrm{~m}$ d'altitude. En Bulgarie, l'espèce serait crénophile.

T. gallicum, dont la distribution dans les Pyrénées a été éludiée par DÉcamPs (1967), présente clle aussi un spectre écologique étendu :

- sources et ruisselets de montagne (entre 1100 et $1600 \mathrm{~m}$ d'altitude), biolopes dans lesquels vivent les populations les plus denses de l'espèce;

- ruisseaux froids de haute altilude (entre 1300 et $2000 \mathrm{~m}$ );

- ruisseaux et torrents à températures variables (entre 500 et $1200 \mathrm{~m}$ );

- déversoirs de lacs (entre 1800 el $2300 \mathrm{~m}$ ), où elle est plus rare.

T. sardoum est bien représentée dans les sources et les petits ruisseaux d'altitude moyenne. On l'y trouve en permanence avec une densité de 10 à 20 larves par $\mathrm{m}^{2}$ et, même, elle pullule au début de l'été dans un petit ruisseau (altitude $450 \mathrm{~m}$ ) où j'ai récolté jusqu'à 90 individus sur une seule pierre. Les larves et nymphes vivent aussi dans des cours d'eau plus importants (rivières) mais leur effectif est toujours modeste (1 à 4 individus par $\left.\mathrm{m}^{2}\right)$.

L'espèce corse, bien que peu alticole, n'est pas une espèce thermophile. Elle termine son évolution préimaginale au début de l'été, les nymphes se formant entre 12 el $17^{\circ} \mathrm{C}$.

\section{5. - Répartition géographique.}

Les Thremma ont une aire de répartition médio-sud européenne, comme on peut le voir sur la carte de distribution des espèces (fig. 11).

L'aire de $T$. gallicum est la plus étendue (sierras centrales d'Espagne, Pyrénées, Massif Central, Forêt Noire). Il semble que l'espèce a évolué, au sein de son aire, en sous-espèces locales (cf. T. gallicum arvernense). Peut-être aux deux extrémités de cette aire existe-t-il une différenciation morphologique comme celle qui sépare la forme des Pyrénées de celle du Massif Central?

T. anomalum se localise dans la partie orientale des Balkans : Massif du Banal (Roumanie), Serbie et Macédoine (Yougoslavie), monts Rhodope (Bulgarie), Grèce.

T. sardoum est endémique dans le système insulaire cyrnosardc. Il semble que le genre soit présent également en Afrique du Nord, puisque Lestage (1925) a signalé, en Algérie, la présence de larves de Thremma, sans qu'il ait pu leur donner une dénomination spécifique, Compte tenu de cette dernière localisa- 


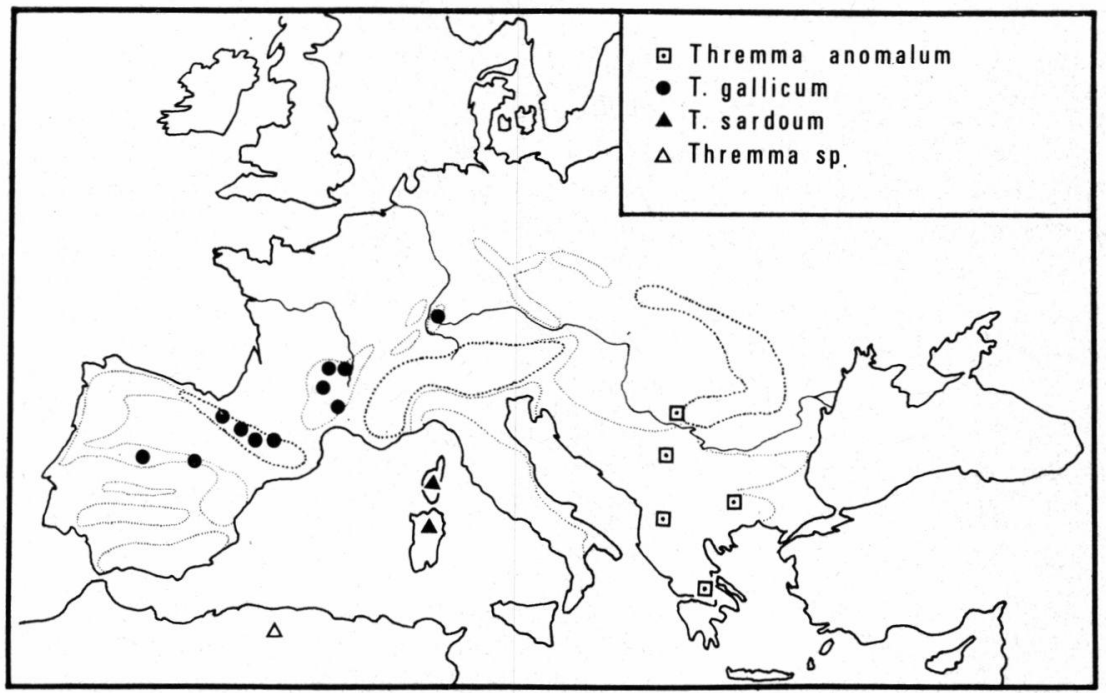

FIG. 11. - Distribution géographique du genre Thremma.

tion, le genre Thremma apparaît plutôt comme une lignée circum-méditerranéenne, probablement apparue à une époque où la Méditerranée occidentale constituait une terre émergée faisant partie intégrante du continent européen.

Le genre, compte tenu, d'une part, de son isolement systématique parmi les Trichoptères, d'autre part, de sa localisation dans les vieux massifs hercyniens et de sa répartition discontinue au sein d'une aire circumméditerranéenne et médio-européenne, apparaît comme le représentant actuel d'une vieille lignée secondaire ou tertiaire. On doit le considérer comme un témoin de la faune autochtone qui vivait dans les eaux courantes d'Europe occidentale et méridionale au secondaire et au tertiaire.

\section{RÉSUMÉ}

Thremma gallicum comprend en France deux populations morpholo. giquement distinctes; l'une vit dans le Massif Central, l'autre dans les Pyrénées. La population du Massif Central représente une nouvelle sousespèce : $T$. gallicum arvernense.

L'auteur donne la première description de la larve de $T$, sardoum et complète la connaissance de l'imago de cette espèce.

Les larves des trois espèces du genre sont étudiées du point de vue morphologique et écologique.

La répartition géographique des espèces suggère une grande ancienneté pour le genre Thremma. 


\section{ABOUT THE GENUS THREMMA}

[Trichoptera, Thremmatidac]

In France, Thremma gallicum includes two morphologically different populations; one lives in the Massif Central, the other in the Pyrenees. The population from Massif Central represents a new sub-species : T. gallicum arvernense.

The autor gives the first description of the larva of $T$. sardoum as well as notes on the imago.

The morphology and ecology of the larvae of the three species of this genus are also studied.

The geographical distribution of the species indicates that the genus Thremma is very ancient.

\section{MONOGRAPHE DER GATTUNG THREMMA}

[Trichoptera, Thremmatidae]

Thremma gallicum umfasst in Frankreich zwei morphologisch verschiedene Populationen. Eine kommt im Zentral Massiv vor, die andere in den Pyrenäen. Die Population des Zentral Massives ist eine neue Unterart : $T$. gallicum arvernense.

Der Autor beschreibt erstmals die Larve von $T$. sardoum und vervollständigt die Kenntnis der Imago dieser Art.

Die Larven der drei Arten der Gattung werden morphologisch untersucht. Die geographische Verbreitung der Arten lässt auf ein hohes phylogenetisches Art des Genus Thremma schliessen.

\section{TRAVAUX GITÉS}

Botosaneanu (L.). 1956. - Recherches sur les Trichoptères de Bulgarie recueillis par MM. les Prof. A. Valkanov et B. Rusev. Beitr. z. Entom., 6, (3-4) : 354-402.

Botosaneanu (I.). 1957. - Recherches sur les Trichoptères (imagos) de Roumanie. Bull, entom. Pologne, 26 : 384-433.

Botosaneanu (L.). 1960. - Sur quelques régularités observées dans le domaine de l'écologie des insectes aquatiques. Arch. Hydrobiol., 56 (4) : $370-377$.

Costa (A.). 1884. - Notizie ed Osservazioni sulla Geofauna sarda -Memoria III. Ann. Real. Accad. Sci. Napoli, $1\left(2^{\circ} \mathrm{S}\right)$ : 7-32.

Decamps (H.. 1967. - Ecologie des Trichoptères de la vallée d'Aure (Hautes-Pyrénées). Ann, Limnol., 3 (3) : 399-577.

GiUdigeli. (J.). 1968. - Recherches sur le peuplement, l'écologie et la biogéographic d'un réseau hydrographique de la Corse centrale. Thèse, Fac. Sci. Marseille : 437 p.

Jacquemart (S.) et Colneau (Y.). 1966. - A propos de Thremma gallicum Mac Lachlan (Trichoptera). Bull. Inst. r. Sci. nat. Belg., 42 (17) : 1-14.

Kiapalek (F.). 1908. - Larva a pouzdro Thremma gallicum Mc Lachl. Acta Soc. entom. Bohem., 5, p. 276.

MaC IAACHLAN (R.). 1874-1884. - A monographic revision and synopsis of the Trichoptera of the european fauna, $523 \mathrm{p.}$, pl. : 1-51. First additional suppl. 76 p., 7 pl. London.

Mosely (M.E.). 1930. - Corsican Trichoptera. Eos, 6 : 147-184. 